\title{
Editorial
}

\section{Adaptive Impact Absorption}

\author{
Jan Holnicki-Szulc, ${ }^{1}$ Mohamed Ichchou, ${ }^{2}$ Zhongdong Duan, ${ }^{3}$ and Lukasz Jankowski ${ }^{1}$ \\ ${ }^{1}$ Institute of Fundamental Technological Research (IPPT PAN), Ulica Pawińskiego 5B, 02-106 Warsaw, Poland \\ ${ }^{2}$ École Centrale de Lyon, 36 avenue Guy de Collongue, 69134 Ecully Cedex, France \\ ${ }^{3}$ Shenzhen Graduate School of Harbin Institute of Technology, Xili, Shenzhen University Town, Shenzhen 518055, China \\ Correspondence should be addressed to Jan Holnicki-Szulc; holnicki@ippt.pan.pl
}

Received 10 December 2015; Accepted 14 December 2015

Copyright (c) 2016 Jan Holnicki-Szulc et al. This is an open access article distributed under the Creative Commons Attribution License, which permits unrestricted use, distribution, and reproduction in any medium, provided the original work is properly cited.

The surging quest for safety is a clearly visible trend in modern societies. One of the related areas of research is the design of systems protecting against heavy dynamic loads such as low and medium velocity traffic-related impacts and environmental loadings. Commonly applied passive systems are typically designed to withstand a specified, well-defined heavy load scenario, which limits their performance over any wider range of loads, including the less heavy loads that are encountered in the lifetime of a typical structure much more often than the maximum limiting loads. As an example, such a situation is particularly evident in the case of aircraft landing gears, which are required by the regulations to be designed for the maximum specified touchdown load, which almost never occurs in practice. As a result, a typical landing gear during its lifetime operates constantly in a nonoptimized mode and transfers to the fuselage much higher loads than it would be technically possible with a proper adaptation [1]. Another illustrative example is provided by the traditionally rigid support structures of railway carriages: as demonstrated in [2], their crashworthiness can be significantly improved by employing controllable adaptive members. In these and many other applications, embodying structures with a certain amount of intelligence, understood here as the interconnected abilities of sensing (real-time recognition and identification of the loading conditions) and optimum self-adaptation, can considerably increase dissipation of load energy, reduce operational loads transferred to the structure, and thus contribute to limiting the risk of structural failure or the fatigue factor.

Basically, the related idea of optimum counteraction to the excitation is one of the fundamental concepts behind many successful applications of the control theory, such as piezo-based damping of space structures [3]. However, typical formulations lead to problems of active structural control, which require the actuators to be able to generate and transfer to the structure significant external loads. Although effective, such an approach requires high energy supply, and in case of power or hardware failures it brings the risk of dangerous instabilities, which needs to be separately addressed. These shortcomings of active control approaches can be significantly reduced by implementing semi-active control strategies and adaptive impact absorption (AIA) systems. The general concept of an AIA system, considered for the first time probably in [4], refers to an online adaptation of an energy-absorbing structure to an extreme overloading by

(1) real-time recognition of an imminent impact load and identification of its crucial characteristics,

(2) application of semi-actively controllable dissipaters of a various nature (magnetorheological fluids, piezoactuated devices, pneumatic systems, etc.) in order to optimally dissipate the energy of the impact.

Such an approach allows the structural capacity for absorption of unexpected extreme loads to be significantly enlarged. Good examples of potential practical applications for AIA systems are adaptive road barriers, adaptive landing gears, and adaptive airbags for emergency landing scenarios.

The field of AIA generates a number of original research problems related to the optimum design of AIA systems, real-time impact load identification, globally optimum semiactive control, local modelling of the involved dissipaters, and so forth. This special issue is focused on mathematical modeling and optimization of AIA systems considered in 
their entireties and at the level of their adaptive components. Three of the submitted papers investigate the problems related to the global design and control of AIA systems. J. Szklarski and M. Wiklo demonstrate in their contribution "Designing of Elastoplastic Adaptive Truss Structures with the Use of Particle Swarm Optimization" the applicability and effectiveness of a heuristic algorithm of particle swarm optimization (PSO) to the problem of global design of an adaptive impact-absorbing structure, including placement of adaptive fuses and redistribution of mass and yield stress limits. In comparison to the classical gradient-based optimization, the particularly appealing feature of PSO is its ability to provide alternative and equally good solutions, as well as its flexibility with respect to the definition of the objective function. In the paper titled "Prestress Accumulation-Release Technique for Damping of Impact-Born Vibrations: Application to SelfDeployable Structures," A. Mróz et al. study an interesting adaptive technique (prestress accumulation-release, PAR) intended for damping of impact-born vibrations of frame structures. The crucial point providing for its effectiveness is its ability to convert the vibration energy from lightly damped, lower order vibration modes into high-order modes, which are highly damped by means of standard mechanisms of material damping. Z. Wang et al. consider in the contribution "Impact Safety Control Strategy for the Battery System of Electric Bus" an important practical application and develop a control strategy for a battery system in an electric bus to ensure high-voltage safety during side impacts in traffic conditions.

The other group of papers in this special issue focuses on local modelling of actuators dedicated to AIA applications. In the paper "Pneumatic Adaptive Absorber: Mathematical Modelling with Experimental Verification," G. Mikułowski and R. Wiszowaty propose, study, and experimentally validate a mathematical model of a pneumatic adaptive absorber. An important advantage of the proposed solution is its double-chamber, closed design, which allows the device to be used repetitively without reinflation. Finally, in the contribution "Crashworthiness of Inflatable Thin-Walled Structures for Impact Absorption," C. Graczykowski and J. HolnickiSzulc investigate the crashworthiness of inflatable thin-walled structures in terms of their local response, as well as in terms of their influence on the global structural response. The adaptive control is implemented in two ways: the preimpact inflation to a desired pressure and then a gradual release of the pressure during impact reception.

Compared to passive impact-handling systems, the significantly better performance of AIA systems can be attributed to the paradigm of smart self-adaptivity, which is ubiquitous in nature, but still sparsely applied in structural and transport engineering. We hope that this special issue, by stimulating a concerted effort in facing a number of related research challenges, will contribute to their ultimate success: the dream of structural safety coming true.

\section{Acknowledgments}

We would like to thank all the authors for supporting this special issue with their excellent contributions. We express also our appreciation to all the reviewers for their insightful and constructive comments. Financial support of the Polish National Science Centre (Project AIA, DEC2012/05/B/ST8/02971) is gratefully acknowledged.

Jan Holnicki-Szulc
Mohamed Ichchou
Zhongdong Duan
Łukasz Jankowski

\section{References}

[1] G. Mikułowski and Ł. Jankowski, "Adaptive landing gear: optimum control strategy and potential for improvement," Shock and Vibration, vol. 16, no. 2, pp. 175-194, 2009.

[2] J. Holnicki-Szulc and L. Knap, "Adaptive crashworthiness concept," International Journal of Impact Engineering, vol. 30, no. 6, pp. 639-663, 2004.

[3] A. Preumont, J.-P. Dufour, and C. Malekian, "Active damping by a local force feedback with piezoelectric actuators," Journal of Guidance, Control, and Dynamics, vol. 15, no. 2, pp. 390-395, 1992.

[4] J. Holnicki-Szulc, A. Maćkiewicz, and P. Kołakowski, "Design of adaptive structures for improved load capacity," AIAA Journal, vol. 36, no. 3, pp. 471-476, 1998. 


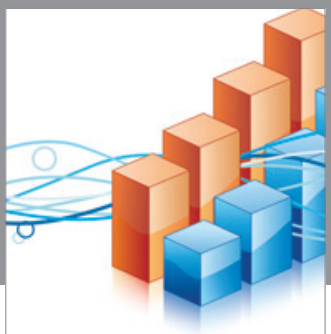

Advances in

Operations Research

vatem alat4

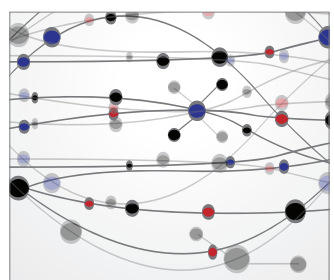

\section{The Scientific} World Journal
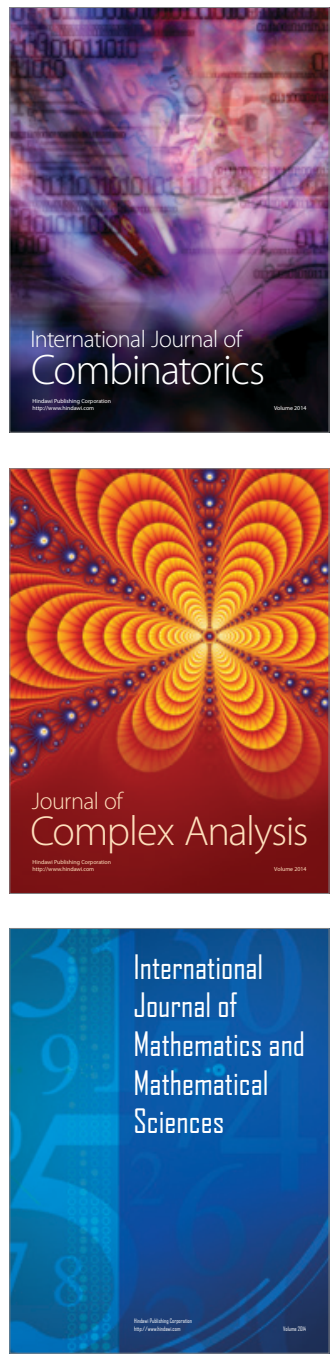
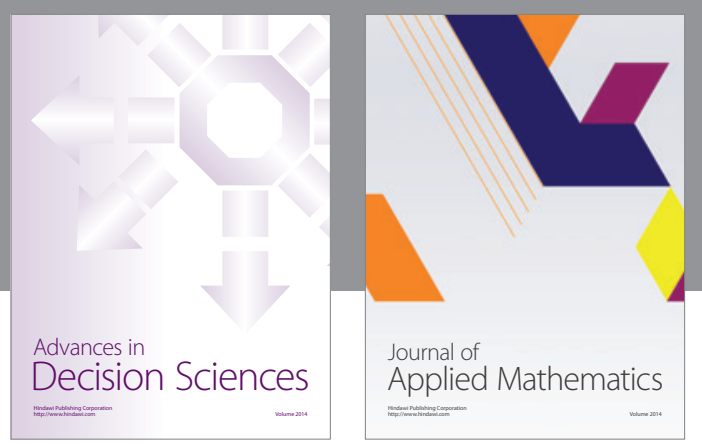

Algebra

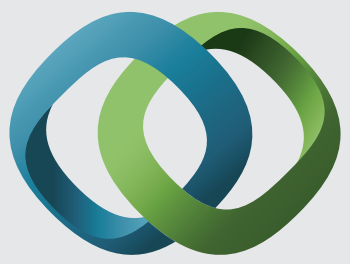

\section{Hindawi}

Submit your manuscripts at

http://www.hindawi.com
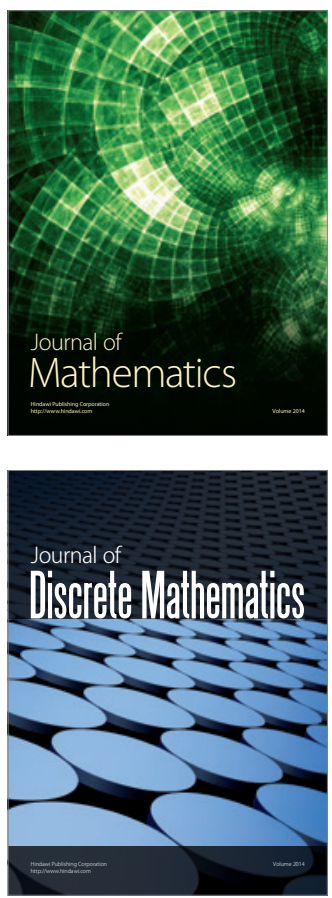

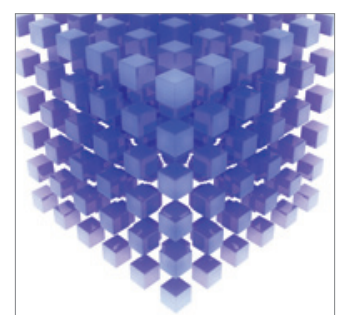

Mathematical Problems in Engineering
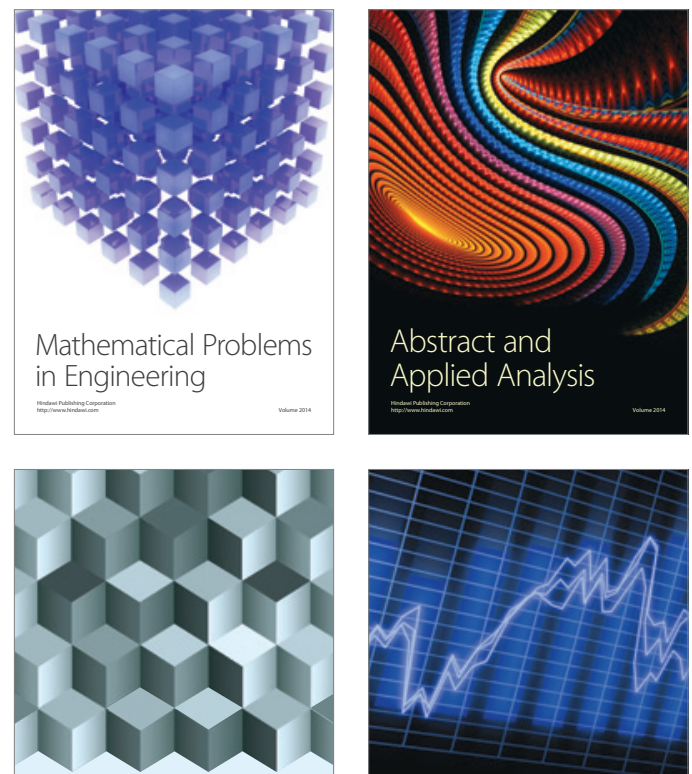

Journal of

Function Spaces

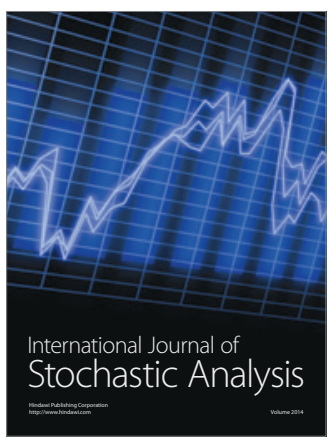

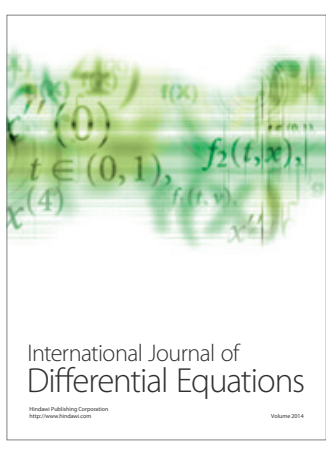
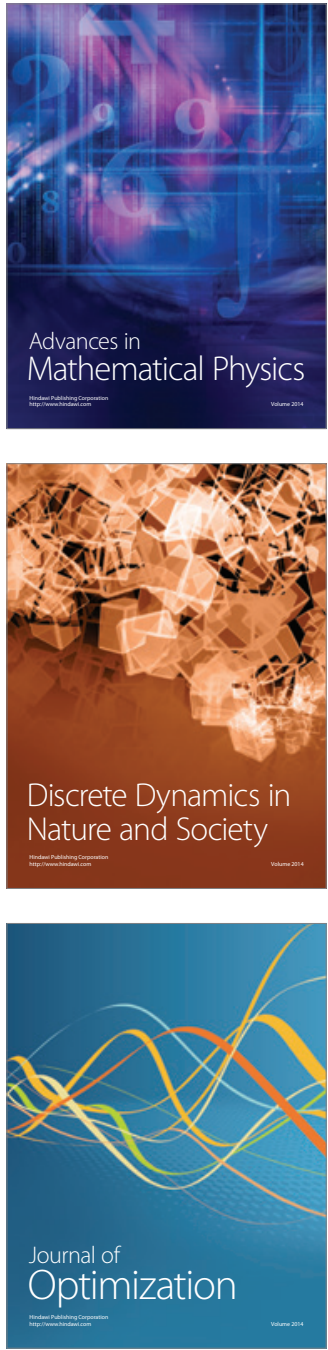